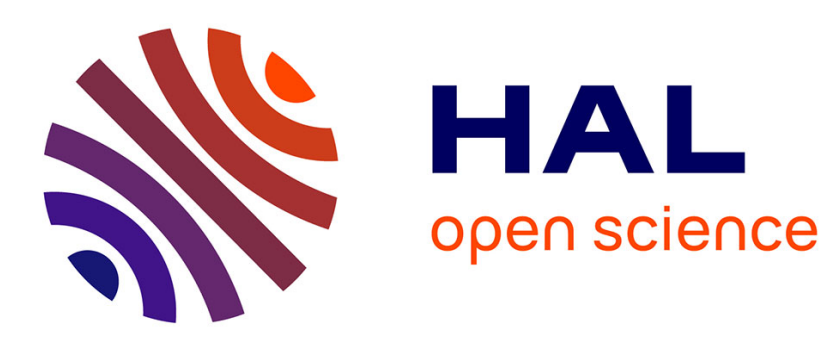

\title{
Localization of Spatially Distributed Near-Field Sources with Unknown Angular Spread Shape
} Jad Abou Chaaya, José Picheral, Sylvie Marcos

\section{To cite this version:}

Jad Abou Chaaya, José Picheral, Sylvie Marcos. Localization of Spatially Distributed NearField Sources with Unknown Angular Spread Shape. Signal Processing, 2015, 106, pp.259-265. 10.1016/j.sigpro.2014.07.007 . hal-01231774

\section{HAL Id: hal-01231774 https://hal.science/hal-01231774}

Submitted on 20 Nov 2015

HAL is a multi-disciplinary open access archive for the deposit and dissemination of scientific research documents, whether they are published or not. The documents may come from teaching and research institutions in France or abroad, or from public or private research centers.
L'archive ouverte pluridisciplinaire HAL, est destinée au dépôt et à la diffusion de documents scientifiques de niveau recherche, publiés ou non, émanant des établissements d'enseignement et de recherche français ou étrangers, des laboratoires publics ou privés. 


\title{
Localization of Spatially Distributed Near-Field Sources with Unknown Angular Spread Shape
}

\author{
Jad ABOU CHAAYA ${ }^{\mathrm{a}, \mathrm{b}, *}$, Jose PICHERAL ${ }^{\mathrm{a}}$, Sylvie MARCOS ${ }^{\mathrm{b}}$ \\ ${ }^{a}$ Department of Signal Processing and Electronic Systems, Supelec, 91192 GIF-sur-Yvette, France. \\ ${ }^{b}$ Laboratoire des signaux et systèmes, CNRS, Supelec, 91192 GIF-sur-Yvette, France.
}

\begin{abstract}
In this paper, we propose to localize and characterize coherently distributed (CD) sources in near-field. Indeed, it appears that in some applications, the more the sources are close to the array of sensors, the more they can seem scattered. It thus appears of the biggest importance to take into account the angular distribution of the sources in the joint direction of arrival (DOA) and range estimation methods. The methods of the literature which consider the problem of distributed sources do not handle with the case of near field sources and require that the shape of the dispersion is known. The main contribution of the proposed method is to estimate the shape of the angular distribution using an additional shape parameter to address the case of unknown distributions. We propose to jointly estimate the DOA, the range, the spread angle and the shape of the spread distribution. Accurate estimation is then achieved even when the shape of the angular spread distribution is unknown or imperfectly known. Moreover, the proposed estimator improves angular resolution of the sources.
\end{abstract}

Keywords: Array signal processing, Source localization, Distributed sources, Near-field, Angular spread shape estimation.

\section{Introduction}

In array signal processing, most of the algorithms that estimate the direction of arrival (DOA) have been developed on the assumption of point emitting sources in far-field. This modeling assumption is not suitable for several physical examples. Indeed in many applications such as wireless communications, radar, sonar or localization of acoustic sources, the angular spread of the spatial extension cannot be ignored. One can cite as a motivating subject for future study, the localization of aero-acoustic sources on the body of a car [1]. The purpose of such an application is to allow car manufacturers to improve passenger comfort by reducing aerodynamic noise.

Two models are classically used in the literature for spatial distributed sources. In telecommunications, the model considers independent local scatterers around the source [2]. A second widely considered model [3] assumes that the source is continuously spatially extended around a nominal DOA with an angular spread distribution. The present paper is based on the second

\footnotetext{
${ }^{*}$ Corresponding author

Email addresses: jad.abouchaaya@supelec.fr (Jad ABOU CHAAYA), jose.picheral@supelec.fr (Jose PICHERAL), sylvie.macros@lss.supelec.fr (Sylvie MARCOS)

Preprint submitted to Signal Processing 
model. In order to estimate both the DOA and the angular spread of the distributed sources in the far-field case, Valaee proposed the Distributed Signal Parameter Estimator (DSPE) [3] which is a MUSIC-like algorithm [4]. However, the assumption of knowing the distribution function of the spatial extension is not realistic in practice. Besides, such a method suffers from robustness issues with respect to the imperfect knowledge of the angular spread distribution. In other words, an a priori information about the angular spread shape must be provided for good performance. A solution based on decoupling the DOA $\theta$ from the angular spread $\Delta$ estimation have been proposed in [5] using the covariance matching method. This method is developed in the case of Incoherently Distributed ${ }^{1}$ (ID) sources but suffers from ambiguity problem. Zoubir and Wang proposed in [6], a decoupled estimation of the DOA and the angular spread distribution for far-field sources. This method provides a robust estimation only for DOA but it requires the knowledge of the angular spread shape distribution to estimate the angular spread parameter.

Near-field sources localization has been an active field of research for many years $[7,8,9,10$, 11]. As far as we know, the estimators proposed in the literature do not take into consideration the aspect of spatial distributed sources in conjunction with the aspect of near-field propagation. However, for a given physical extension of the source, the angular spread becomes more relevant as the source gets closer to the array.

These reasons motivate us to propose an estimator of CD sources in near-field which is robust to the imperfect knowledge of the angular spread distribution. First, we introduce the model [3] in the near-field context with the aim of considering the angular spread when sources are close to the array. Then, we discuss limits of DSPE algorithm with different angular distribution shapes for the near-field. Finally, we propose a modification of the DSPE which consists in using a generic function family to describe the angular spread distribution. The method called Joint Angle, Distance, Spread, Shape Estimator (JADSSE) consists in the estimation of the distribution shape parameter in addition to the three other parameters.

The contribution is organized as follows. In section 2, we extend the model for distributed sources located in near-field. In section 3, we present the generalization of the DSPE and the new JADSSE method. In section 4, the performance of the proposed algorithms are compared by numerical simulations. Finally we present the conclusions.

\section{Signal model}

Let us assume $q$ spatially distributed narrow-band near-field sources impinging on a Uniform Linear Array (ULA) of $M$ sensors. The distance between two adjacent sensors is $d=\lambda / 2$, where $\lambda$ is the wavelength of the signals. We also suppose that the sources and the sensors are in the same plane. The $M \times 1$ baseband signal vector measured by the $M$ sensors is given by $\underline{\mathbf{x}}(t)=\left[x_{1}(t), \ldots, x_{M}(t)\right]^{T}$, where $(.)^{T}$ is the transpose operator. Authors in [3] proposed a model for the distributed sources in far-field. We here extend this model in the near-field context, considering an angular spread of $\mathrm{DOA}^{2}$ :

$$
\underline{\mathbf{x}}(t)=\sum_{i=1}^{q} \int_{-\frac{\pi}{2}}^{\frac{\pi}{2}} \underline{\mathbf{a}}\left(\phi, r_{i}\right) v_{i}\left(\phi, \theta_{i}, \Delta_{i}, t\right) d \phi+\underline{\mathbf{n}}(t) .
$$

\footnotetext{
${ }^{1}$ Signals arriving from multiple directions are assumed to be uncorrelated

${ }^{2}$ Similarly a range spread can be also considered, however, in order to make the paper more readable we only consider the angular spread
} 
$v_{i}\left(\phi, \theta_{i}, \Delta_{i}, t\right)$ denotes the signal angular distribution for the $i$-th source. $\theta_{i}$ is the central DOA for the source and $\Delta_{i}$ is the angular spread. $\underline{\mathbf{n}}(t)$ is the $M \times 1$ white additive noise vector. The $M \times 1$ vector $\underline{\mathbf{a}}(\phi, r)$ represents the near-field array response for a source located in direction $\phi$ and at range $r$.

In the near-field context, the signal propagation time between sensor $m$ and a reference sensor (e.g the first one) can be approximated ${ }^{3}$ (see [12] for details). In this case, the $(m+1)$-th element of the steering vector $\underline{\mathbf{a}}(\phi, r)$ is expressed as:

$$
a_{m+1}(\phi, r)=\exp \left(-j \pi \sin (\phi) m+j \frac{\pi \lambda}{4 r} \cos ^{2}(\phi) m^{2}\right) .
$$

Throughout the paper, we consider that the sources are coherently distributed (CD). A CD source is described by a temporally invariant angular distribution and the components of the signal are fully correlated for the whole angular spread (for more details see [3]). Thus, the signal angular distribution for the $i$-th CD source can be expressed by:

$$
v_{i}\left(\phi, \theta_{i}, \Delta_{i}, t\right)=s_{i}(t) h_{i}\left(\phi, \theta_{i}, \Delta_{i}\right)
$$

where $s_{i}(t)$ is a random complex signal emitted by the $i$-th source and $h_{i}\left(\phi, \theta_{i}, \Delta_{i}\right)$ represents a deterministic angular spread distribution. For multiple CD sources, the model of the received signal for $q$ sources in near-field is given by:

$$
\begin{array}{r}
\underline{\mathbf{x}}(t)=\sum_{i=1}^{q} s_{i}(t) \underline{\mathbf{c}}\left(\theta_{i}, \Delta_{i}, r_{i}\right)+\underline{\mathbf{n}}(t), \\
\underline{\mathbf{c}}\left(\theta_{i}, \Delta_{i}, r_{i}\right)=\int_{-\frac{\pi}{2}}^{\frac{\pi}{2}} \underline{\mathbf{a}}\left(\phi, r_{i}\right) h_{i}\left(\phi, \theta_{i}, \Delta_{i}\right) d \phi,
\end{array}
$$

$\underline{\mathbf{c}}\left(\theta_{i}, \Delta_{i}, r_{i}\right)$ is the vector obtained by integrating the steering vector $\mathbf{a}\left(\phi, r_{i}\right)$ with the angular distribution of the $i$-th source $h_{i}\left(\phi, \theta_{i}, \Delta_{i}\right)$. The source signal and noise time samples are modeled by random, complex, centered and independent processes. We assume that the noise and the sources $v_{i}\left(\phi, \theta_{i}, \Delta_{i}, t\right)$ are uncorrelated with each other. Considering the previous assumptions, the correlation matrix of the array output is given by:

$$
\mathbf{R}_{x x}=E\left[\underline{\mathbf{x}}^{H}\right]=\mathbf{C S C}^{H}+\sigma^{2} \mathbf{I},
$$

where $E[$.$] is the statistical expectation operator, \mathbf{C}$ is the $M \times q$ matrix containing the column vectors $\underline{\mathbf{c}}\left(\theta_{i}, \Delta_{i}, r_{i}\right)$ for $i=1, \ldots, q, \mathbf{S}$ is the sources correlation matrix with the $i j$ th component defined as $s_{i j}=E\left[s_{i} s_{j}^{*}\right], \sigma^{2}$ is the noise variance and $\mathbf{I}$ is the $M \times M$ identity matrix. The sources are assumed to be uncorrelated with each other, so that $\mathbf{S}$ is diagonal. The correlation matrix $\mathbf{R}_{x x}$ can be estimated from the $L$ snapshots by:

$$
\hat{\mathbf{R}}_{x x}=\frac{1}{L} \sum_{l=1}^{L} \underline{\mathbf{x}}\left(t_{l}\right) \underline{\mathbf{x}}^{H}\left(t_{l}\right) .
$$

\footnotetext{
${ }^{3}$ Approximation of the propagation time is not necessary to extend the model [3] in the near-field and the propagation time can be also used in its general form.
} 


\section{Proposed estimators}

In this section, we present two methods of joint angle, spread and range estimation for the localization of CD sources in near-field. First, we introduce an extension of the MUSIC-like DSPE algorithm [3], called Near Field-DSPE (NF-DSPE). This method is highly dependent on the shape of $h(\phi, \theta, \Delta)$. Next, we present the Joint Angle, Distance, Spread and Shape Estimator (JADSSE). This algorithm consists of a robust estimation of all the above mentioned parameters to localize the distributed sources in near field. Finally, for comparison we recall the Near Field Estimator (NFE) to localize point sources [13].

\subsection{Near Field-DSPE}

After an eigen analysis, the observation space of $\hat{\mathbf{R}}_{x x}$ can be decomposed into a signal subspace and a noise subspace [4]. The estimated noise subspace basis $\hat{\mathbf{E}}_{n}$ is constructed with the $M-q$ eigenvectors $\underline{\mathbf{e}}_{i}$ associated with the lowest $M-q$ eigenvalues of $\hat{\mathbf{R}}_{x x}$. Exploiting the properties of the eigenvectors leads us to the following equality $\mathbf{C}^{H} \hat{\mathbf{E}}_{n}=0$ [4].

The proposed estimator consists in minimizing the norm of the product between the estimated noise subspace $\hat{\mathbf{E}}_{n}$ and the analysis vector $\underline{\mathbf{c}}^{H}(\theta, \Delta, r)$. To obtain the estimated parameters $\hat{\theta}, \hat{r}$ and $\hat{\Delta}$ for the $q$ sources, the estimator consists in searching the $q$ peaks of the three dimensional cost function $S_{\text {NF-DSPE }}(\theta, \Delta, r)$ :

$$
(\hat{\theta}, \hat{\Delta}, \hat{r})=\underset{\theta, \Delta, r}{\arg \max } S_{\mathbf{N F}-\mathbf{D S P E}}(\theta, \Delta, r)
$$

where:

$$
S_{\mathbf{N F}-\mathbf{D S P E}}(\theta, \Delta, r)=\frac{1}{\underline{\mathbf{c}}^{H}(\theta, \Delta, r) \hat{\mathbf{E}}_{n} \hat{\mathbf{E}}_{n}^{H} \underline{\mathbf{c}}(\theta, \Delta, r)},
$$

and

$$
\underline{\mathbf{c}}(\theta, \Delta, r)=\int_{-\frac{\pi}{2}}^{\frac{\pi}{2}} \underline{\mathbf{a}}(\phi, r) h(\phi, \theta, \Delta) d \phi .
$$

The expression in (8) is a MUSIC criterion with the analysis vector $\mathbf{c}(\theta, \Delta, r)$ which depends on the angular distribution $h(\phi, \theta, \Delta)$. In the literature, commonly used distributions are Gaussian and Uniform. The main drawback of this approach is that the computation of $\underline{\mathbf{c}}(\theta, \Delta, r)$ in (9), requires a priori information about the angular spread distribution $h$.

\subsection{Joint Angle Distance Spread Shape Estimator (JADSSE)}

In this subsection, we aim to localize and characterize CD sources in near-field with various angular spread shapes. Therefore, by adding a shape parameter we propose to use a parameterized functions family $h$ which can adapt to most of well known angular distributions. For instance, we propose to represent $h(\phi, \theta, \Delta)$ by a Raised Cosine (RC) function. This function takes an additional positive parameter $\beta$ into consideration to characterize the shape of the angular spread. The Raised Cosine is expressed by:

$$
h(\phi, \theta, \Delta, \beta)= \begin{cases}1 / \Delta & \text { if }|\phi-\theta| \leq \frac{\Delta}{2}(1-\beta) \\ 1 / 2 \Delta[1+\cos (\gamma)] & \text { if } \frac{\Delta}{2}(1-\beta)<|\phi-\theta| \leq \frac{\Delta}{2}(1+\beta) \\ 0 & \text { otherwise }\end{cases}
$$


where $\gamma=\frac{\pi}{\beta \Delta}\left[|\phi-\theta|-\frac{\Delta}{2}(1-\beta)\right]$. This RC function can adapt to various shapes of angular spread distributions: uniform distribution and bell shaped distribution.

It follows that the proposed JADSSE consists in minimizing the norm of the product of $\hat{\mathbf{E}}_{n}$ and the vector $\underline{\mathbf{c}}(\theta, \Delta, r, \beta)$. The estimator relies on the search for the $q$ maxima of the fourdimensional cost function:

$$
(\hat{\theta}, \hat{\Delta}, \hat{r}, \hat{\beta})=\underset{\theta, \Delta, r, \beta}{\arg \max } \frac{1}{\left\|\underline{\mathbf{c}}^{H}(\theta, \Delta, r, \beta) \hat{\mathbf{E}}_{n}\right\|^{2}},
$$

where:

$$
\underline{\mathbf{c}}(\theta, \Delta, r, \beta)=\int_{-\frac{\Delta}{2}(1+\beta)}^{\frac{\Delta}{2}(1+\beta)} \underline{\mathbf{a}}(\phi, r) h(\phi, \theta, \Delta, \beta) d \phi .
$$

The vector $\mathbf{c}(\theta, \Delta, r, \beta)$ is a function of $h(\phi, \theta, \Delta, \beta)$. Choosing this parametric function family ensures the ability to approximately fit functions like Gaussian for $\beta \geq 1$ and Uniform for $\beta=0$.

By introducing a general function family $h(\phi, \theta, \Delta, \beta)$ and by estimating the shape parameter $\beta$, the proposed JADSSE makes it possible to reconstruct the angular distribution without $a$ priori information about its shape. This blind approach allows to increase the robustness of the estimation with respect to the shape of the angular spread distribution.

\subsection{Near Field Estimator}

Assuming point sources, the standard DOA MUSIC estimator [4] can be generalized for Near-field and it consists in searching the $q$ peaks of the two- dimensional cost function $S_{\mathrm{NFE}}(\theta, r)$ :

$$
(\hat{\theta}, \hat{r})=\underset{\theta, r}{\arg \max } \frac{1}{\underline{\mathbf{a}}^{H}(\theta, r) \hat{\mathbf{E}}_{n} \hat{\mathbf{E}}_{n}^{H} \underline{\mathbf{a}}(\theta, r)},
$$

where $\underline{\mathbf{a}}^{H}(\theta, r)$ is defined by equation (2) and $(\hat{\theta}, \hat{r})$ are the DOA and range estimates, respectively.

\subsection{Computational complexity}

The complexity of JADSSE concerns three points. As for the conventional MUSIC algorithm, the eigen decomposition of the $(M \times M)$ covariance matrix of the received signals which is necessary to obtain the noise eigenvectors appearing in equation (10). As for the DSPE, the analysis vector $\mathbf{c}(\theta, \Delta, r, \beta)$ must be computed through an numerical integral. The third point concerns the search of the maxima of $4 \mathrm{D}$ criterion defined in equation (10). JADSSE is more complex than DSPE because of the additional parameters $r$ and $\beta$, which are due to the near-field context and the estimation of the angular distribution shape.

To decrease the computational complexity, methods have been proposed in the literature to decouple the parameter estimation either within the case of near-field sources or within the case of distributed sources. For example, the approach proposed in $[7,8]$ to decouple the estimation of the DOA and range can also be applied to JADSSE. This technique is based on the fact that $\underline{\mathbf{a}}(\phi, r)$ in $(11)$ is a periodic function of the DOA $\phi$. It can be approximated by $\underline{\mathbf{a}}(\phi, r) \approx \mathbf{D}(r) b(\phi)$ (see [7] for details).

Another approach proposed in [6] to the decoupling of the estimation of the DOA and the angular spread can be used to reduce the computational cost of JADSSE. It relies on a first order approximation of the angular spread in the generalized steering vector $\mathbf{c}(\theta, \Delta, r, \beta)$ of equation (11). The analysis vector $\underline{\mathbf{c}}(\theta, \Delta, r, \beta)$ can be approximated by $\underline{\mathbf{c}}(\theta, \Delta, r, \beta) \simeq \boldsymbol{\Phi}\left(\theta_{i}, r_{i}\right) \mathbf{b}\left(\Delta_{i}, \beta_{i}\right)$, where $\boldsymbol{\Phi}\left(\theta_{i}, r_{i}\right)=\operatorname{diag}\left(\mathbf{a}\left(\theta_{i}, r_{i}\right)\right)$ and $\mathbf{b}\left(\Delta_{i}, \beta_{i}\right)$ is a real valued vector that depends on the angular distribution. Using the approximation, we can rewrite the cost function for JADSSE in equation (10) into two 2D optimization problems for DOA estimation. 


\section{Numerical Results}

In this section, we compare the performance of the three methods (JADSSE, NF-DSPE and NFE) presented in section 3. For JADSSE, Raised Cosine angular spread distribution with parameter $\beta$ is used and the NF-DSPE performance is evaluated using three different angular spread distributions : Gaussian, uniform and Butterworth. To investigate the performance of the proposed estimators, the Root Mean Square Error (RMSE) of the estimates is evaluated with a Monte-Carlo simulation of 100 independent runs. The simplex based method is used to find the maximum of the cost function in equations (7), (10) and (12).

The ULA consists of $M=20$ sensors inter-spaced by $d=\lambda / 2$ and the number of samples is 1000 for estimating the covariance matrix in equation (6).

\subsection{Performance versus $S N R$}

In the first simulation, we consider the following multi-sources scenario: two CD sources and one point source in near-field arrive from directions $\theta_{1}=0^{\circ}, \theta_{2}=30^{\circ}, \theta_{3}=60^{\circ}$ with an angular spread $\Delta_{1}=5^{\circ}, \Delta_{2}=8^{\circ}, \Delta_{3}=0^{\circ}$ with distances of $r_{1}=30 \lambda, r_{2}=25 \lambda, r_{3}=30 \lambda$ respectively from the array.

The source at $\theta_{3}=60^{\circ}$ is punctual. The source at $\theta_{1}=0^{\circ}$ is Gaussian $\mathrm{CD}(\mathrm{GCD})$ with the angular spread distribution :

$$
h\left(\phi, \theta_{1}, \Delta_{1}\right)=\frac{1}{\Delta_{1} \sqrt{2 \pi}} \exp \left[-0.5\left(\frac{\phi-\theta_{1}}{\Delta_{1}}\right)^{2}\right] .
$$

The source at $\theta_{2}=30^{\circ}$ is Uniformly CD (UCD), that is

$$
h\left(\phi, \theta_{2}, \Delta_{2}\right)= \begin{cases}\frac{1}{\Delta_{2}} & \text { if }\left|\phi-\theta_{2}\right| \leq \frac{\Delta_{2}}{2} \\ 0 & \text { Otherwise }\end{cases}
$$

Note that the definition of parameter $\Delta$ depends on the shape of the angular spread distribution. For the Raised Cosine function, $\Delta$ is the width of the function at half amplitude whereas for the Gaussian function, it is the standard deviation. Finally, for the bell function with a Butterworth correlation kernel (used in [3]) $\Delta$ is the $-3 \mathrm{~dB}$ extension width.

The RMSE of the DOA $\theta$ and distance $r$ estimates are plotted on Fig. 1a-2a for the GCD source, on Fig. 1b-2b for the UCD source and on Fig. 1c-2c for the point source. Comparing the different estimators, as expected the point source estimator (NFE) presents bad performance for locating distributed sources. Four conclusions can be drawn from these results. Firstly, NF-DSPE performance is highly sensitive to the angular spread distribution. NF-DSPE has the best performance when the distribution used by the estimator is the same as the distribution of the source, but when the distributions are different, performance degrades. Secondly, when the distribution of the source belongs to the RC family, the JADSSE performance reaches these of NF-DSPE using the true distribution (see UCD source on Fig. $1 b$ and Fig. $2 b$ ). This means that estimating the fourth parameter (the shape parameter $\beta$ ) does not penalize the proposed estimator Thirdly, JADSSE provides reliable estimation even when the distribution of the source does not belong to the RC family (see GCD source on Fig. 1a and Fig. 2a). This result shows that JADSSE using an RC family is quite robust to the shape of the angular spread distribution of the source. Fourthly, NF-DSPE (except with Butterworth) and JADSSE performance perfectly fit those of NFE when dealing with the point source (see Fig. 1c and Fig. 2c). It follows that JADSSE becomes particularly more interesting in multi-source scenarios where point and distributed sources with unknown shape distributions are mixed. 


\subsection{Reconstruction of angular shape distribution}

The same three sources scenario as in 4.1 is considered. The true angular spread distribution $h(\phi)$ is plotted (dashed thick line) in Fig. 3 for both CD sources ${ }^{4}$. The estimated distributions $\hat{h}(\phi)$ are also plotted where $\hat{h}(\phi)$ is constructed using the $\hat{\theta}, \hat{\Delta}$ (and $\hat{\beta}$ for JADSSE) estimates at $\mathrm{SNR}=10 \mathrm{~dB}$. Results show that NF-DSPE provides good reconstruction when the used distribution $h(\phi)$ is the same as the actual distribution of the source. In the other cases the estimated distribution is poorly approximated due to the mismodeling error on $h$. On the contrary, JADSSE provides an accurate estimation of the angular spread distribution even for the GCD source (which is not included in the RC function family).

\subsection{Performance versus the angular spread and shape parameters}

In this simulation, one source with a Raised Cosine distribution is considered with $\theta_{1}=10^{\circ}$, $r_{1}=25 \lambda$ and SNR=20dB. Firstly, performances are plotted in Fig. 4a versus the shape parameter $\beta$ with an angular spread fixed at $\Delta=8^{\circ}$. JADSSE keeps almost stable performance as a function of $\beta$. Meanwhile NF-DSPE presents higher RMSE values when a mismatched $h(\phi)$ is used by the estimator. For instance, NF-DSPE with Uniform distribution approaches JADSSE performance for source with small $\beta$ but when $\beta$ increases (corresponding to a source with a bell shape) NFDSPE (Unif) performances decrease. Conversely, the behavior of the NF-DSPE with a Gaussian distribution is better when $\beta$ increases in the RC source.

Secondly, performance is also plotted on Fig. $4 \mathrm{~b}$ versus dispersion $(\Delta)$ of the source for a fixed shape parameter $(\beta=0.5)$. JADSSE exhibits good performance with only a slight increase in RMSE versus the angular spread. Meanwhile, above $\Delta>5^{\circ}$, ignoring the shape of the angular spread distribution dramatically degrades the performance of NFE and NF-DSPE (with mismatch in distribution shapes). More generally, above a given dispersion, the knowledge or an accurate estimation of the distribution shape is essential to obtain good DOA and range estimates. This motivate the use of JADSSE.

\subsection{Performance versus DOA separation}

For this simulation, two uniform coherently distributed sources are considered at $r_{1}=r_{2}=$ $25 \lambda$ with $\Delta_{1}=5^{\circ}, \Delta_{2}=8^{\circ} ; \theta_{1}=0^{\circ}$ and $\theta_{2}=\theta_{1}+\delta_{\theta}$ where $\delta_{\theta}$ is the DOA source separation. The SNR is fixed at $20 \mathrm{~dB}$. The RMSE of the DOA $\theta$ is plotted as a function of the DOA separation $\delta_{\theta}$ in Fig. 5a for the first source and in Fig. 5b for the second source.

The results show that even for a large overlap of both sources for $\delta_{\theta}<10^{\circ}$, the DOAs are accurately estimated by JADSSE, while the NF-DSPE with mismodeling error suffers from low resolution.

On one hand, for the first source $\left(\Delta_{1}=5^{\circ}\right)$ in Fig. 5a and for small DOA separation, only JADSSE provides accurate estimates. Referring to the mono-source case (Fig. 4b), one can notice that for this dispersion $\left(\Delta_{1}=5^{\circ}\right)$, NF-DSPE performances were roughly the same as those of JADSSE. On another hand, for the second source $\left(\Delta_{1}=8^{\circ}\right)$, NF-DSPE fails to accurately estimate the DOA, even for large separation of the DOA's due to the large dispersion of the source (as seen in the previous mono-source experiment (Fig. 4b)).

\footnotetext{
${ }^{4}$ The third punctual source at $\theta_{3}=60^{\circ}$ is not plotted since the true angular distribution is a Dirac with infinite value, and estimated distributions have too high amplitude to be plotted on the same figure
} 


\section{Conclusion}

In this paper, we propose the JADSSE algorithm for the localization of coherently distributed (CD) sources in near-field. It jointly estimates the angle, the distance and the angular spread with an additional shape parameter. Simulation results have shown that NF-DSPE is limited to the case where the angular spread distribution is known. Meanwhile, JADSSE accurately localizes sources even when $h$ imperfectly fits the Raised Cosine family. The proposed estimator JADSSE also shows good asymptotic performance when the angular function $h$ belongs to the Raised Cosine family. The practical interest of JADSSE can be summarized by: i) its ability to deal with an unknown angular spread distribution; ii) its robustness to various shapes of the angular distribution (RC, Gaussian, uniform and punctual) and iii) its improved characterization of the source by providing an estimate of the angular spread distribution (and the shape of the source). Further studies will focus on the investigation of the JADSSE approach for the real data application presented in the introduction. Indeed, in the aero-acoustic source localization context, the near-field sources present a spatial extension without information regarding its shape.

\section{References}

[1] C. E. Kassis, J. Picheral, Wideband zero-forcing MUSIC for aeroacoustic sources localization, in: Proceedings of the 20th European Signal Processing Conference, 2012, pp. 2283-2287.

[2] T. Trump, B. Ottersten, Estimation of nominal direction of arrival and angular spread using an array of sensors, Signal Processing 50 (12) (1996) 57-69.

[3] S. Valaee, B. Champagne, P. Kabal, Parametric localization of distributed sources, IEEE Transactions on Signal Processing 43 (9) (1995) $2144-2153$.

[4] R. Schmidt, Multiple emitter location and signal parameter estimation, IEEE Transactions on Antennas and Propagation 34 (3) (1986) $276-280$.

[5] O. Besson, P. Stoica, Decoupled estimation of DOA and angular spread for a spatially distributed source, IEEE Transactions on Signal Processing 48 (7) (2000) 1872-1882.

[6] A. Zoubir, Y. Wang, Efficient DSPE algorithm for estimating the angular parameters of coherently distributed sources, Signal Processing 88 (4) (2008) 1071-1078.

[7] A. Weiss, B. Friedlander, Range and bearing estimation using polynomial rooting, IEEE Journal of Oceanic Engineering 18 (2) (1993) 130-137.

[8] D. Starer, A. Nehorai, Passive localization of near-field sources by path following, IEEE Transactions on Signal Processing 42 (3) (1994) 677-680.

[9] N. Yuen, B. Friedlander, Performance analysis of higher order ESPRIT for localization of near-field sources, IEEE Transactions on Signal Processing 46 (3) (1998) 709 -719.

[10] J. Chen, R. Hudson, K. Yao, Maximum-likelihood source localization and unknown sensor location estimation for wideband signals in the near-field, IEEE Transactions on Signal Processing 50 (8) (2002) 1843-1854.

[11] J. Liang, X. Zeng, B. Ji, J. Zhang, F. Zhao, A computationally efficient algorithm for joint range-DOA-frequency estimation of near-field sources, Digital Signal Processing 19 (4) (2009) 596-611.

[12] E. Grosicki, K. Abed-Meraim, Y. Hua, A weighted linear prediction method for near-field source localization, IEEE Transactions on Signal Processing 53 (10) (2005) 3651 - 3660.

[13] Y.-D. Huang, M. Barkat, Near-field multiple source localization by passive sensor array, IEEE Transactions on Antennas and Propagation 39 (7) (1991) 968-975. 


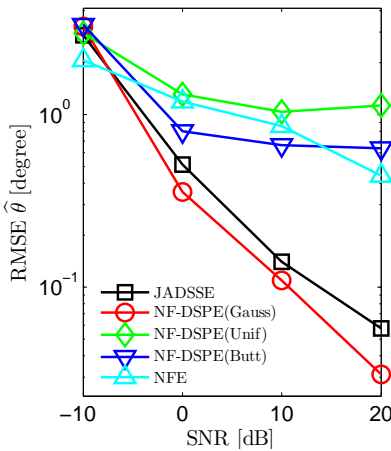

(a) RMSE of $\hat{\theta}_{1}$ for GCD source. (b) RMSE of $\hat{\theta}_{2}$ for UCD source.

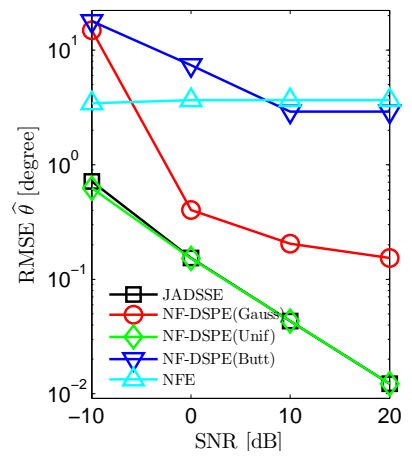

sour

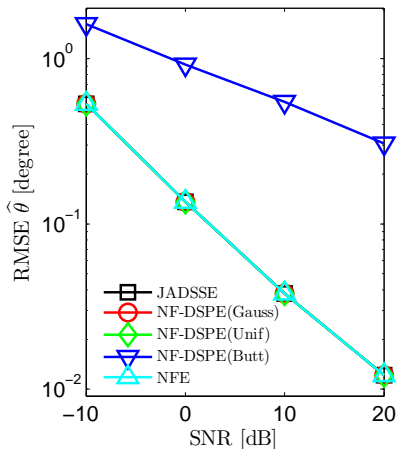

(c) RMSE of $\hat{\theta}_{3}$ for punctual source.

Figure 1: Root Mean Square Error of the DOA parameter $\hat{\theta}$ versus SNR for the 3 sources. True DOAs $\theta_{1}=0^{\circ}, \theta_{2}=30^{\circ}$ and $\theta_{3}=60^{\circ}$. 


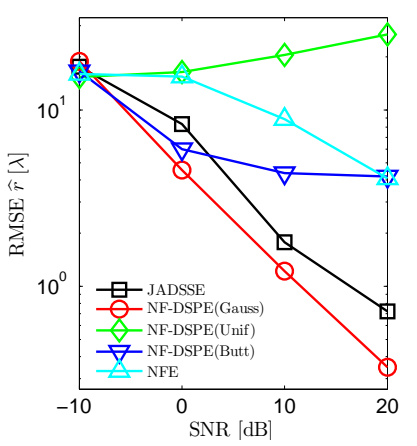

(a) RMSE of $\hat{r}_{1}$ for GCD source. (b) RMSE of $\hat{r}_{2}$ for UCD source.

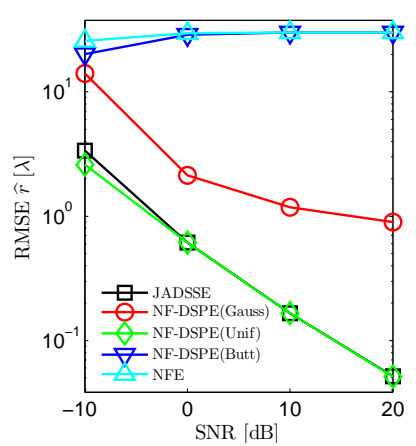

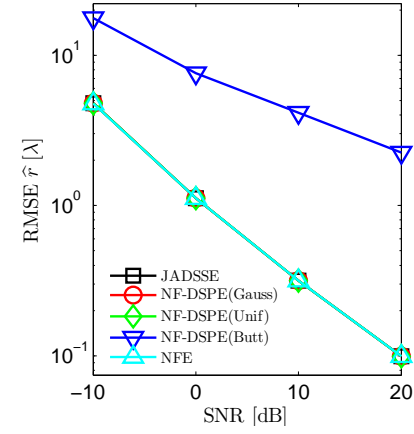

(c) RMSE of $\hat{r}_{3}$ for punctual source.

Figure 2: Root Mean Square Error of the distance parameter $\hat{r}$ versus SNR for the 3 sources. True distances $r_{1}=30 \lambda$, $r_{2}=25 \lambda$ and $r_{3}=30 \lambda$. 


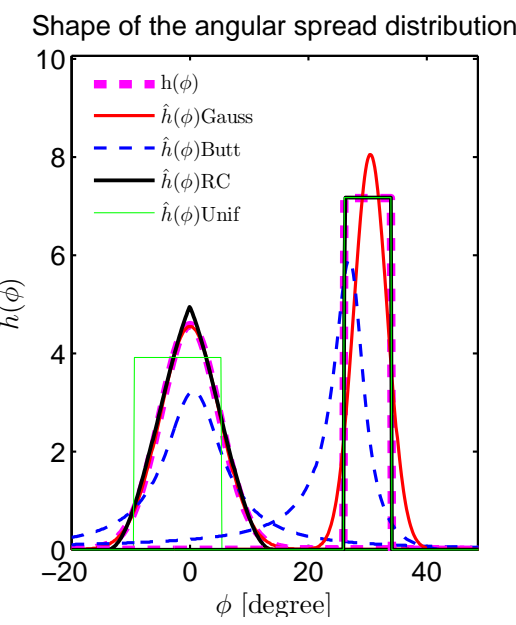

Figure 3: Reconstruction of the angular spread distribution $\hat{h}(\phi)$. 


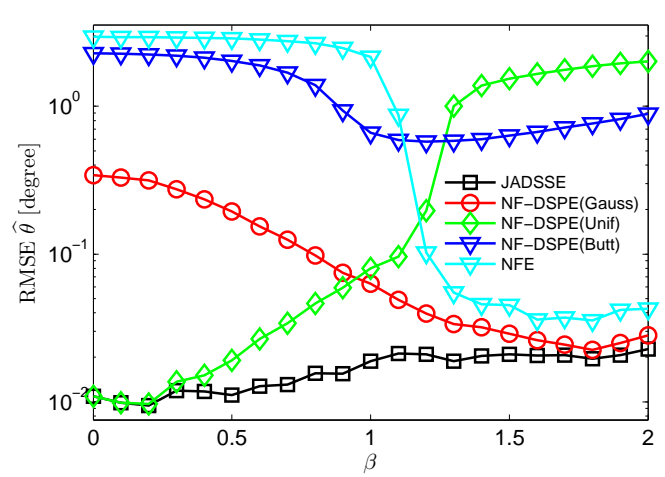

(a) RMSE versus $\beta$ for a fixed $\Delta=8^{\circ}$.

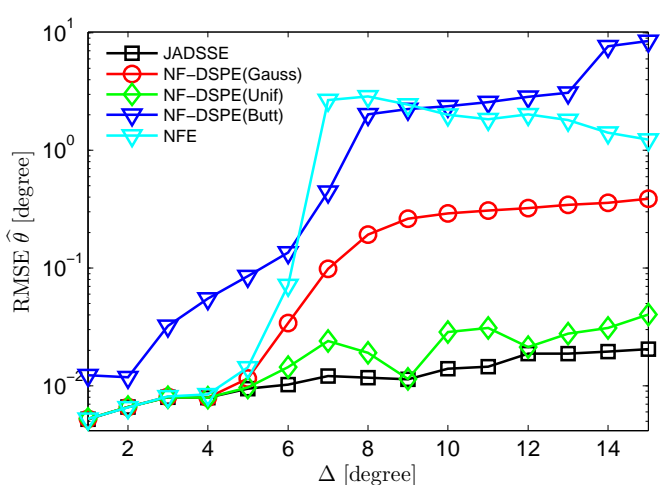

(b) RMSE versus $\Delta$ for a fixed $\beta=0.5$.

Figure 4: Root Mean Square Error of DOA $\hat{\theta}$ for Raised Cosine source at $\mathrm{SNR}=20 d B$. True parameters $\theta=10^{\circ}$ and $r=25 \lambda$. 


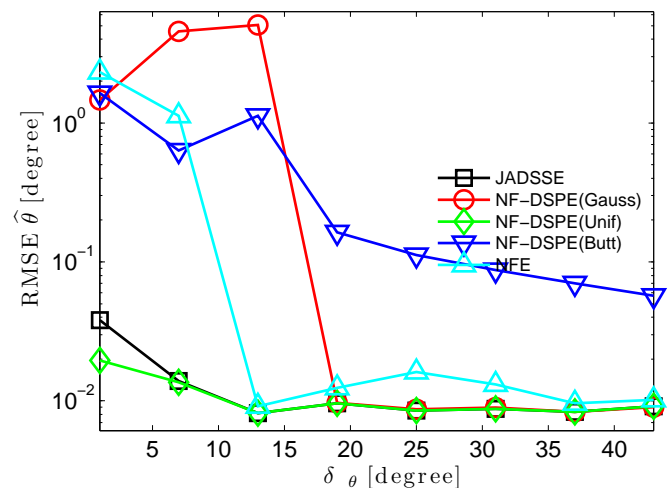

(a) RMSE for UCD source with $\Delta_{1}=5^{\circ}$.

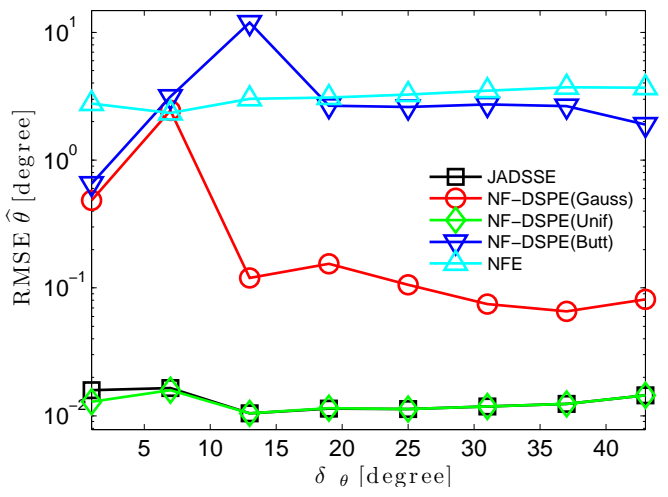

(b) RMSE for UCD source with $\Delta_{2}=8^{\circ}$.

Figure 5: Root Mean Square Error of DOA $\hat{\theta}$ versus $\delta_{\theta}$ for both UCD sources at SNR=20dB. True parameters $\theta_{1}=0^{\circ}$ $\theta_{2}=\theta_{1}+\delta_{\theta}, \Delta_{1}=5^{\circ}, \Delta_{2}=8^{\circ}$ and $r_{1}=r_{2}=25 \lambda$. 